\title{
Percepção do peso corporal acima do ideal, perfil antropométrico e estilo de vida em adolescentes de Recife, PE, Brasil
}

\author{
Perception of body weight above the ideal, anthropometric profile \\ and lifestyle among adolescents in the city of Recife, Brazil
}

\begin{abstract}
Maria José Laurentina do Nascimento Carvalho (https://orcid.org/0000-0002-6705-165X) ${ }^{1}$
Poliana Coelho Cabral (https://orcid.org/0000-0002-2709-4823) ${ }^{1}$

Juliana Souza Oliveira (https://orcid.org/0000-0003-1449-8930) ${ }^{2}$

Pedro Israel Cabral de Lira (https://orcid.org/0000-0002-1534-1620) ${ }^{1}$

Maria Izabel Siqueira de Andrade (https://orcid.org/0000-0003-1087-1320) ${ }^{1}$

Vanessa Sá Leal (https://orcid.org/0000-0001-9492-2580) ${ }^{2}$

Leopoldina Augusta Souza Sequeira-de-Andrade (https://orcid.org/0000-0003-0689-5522) ${ }^{1}$

Fernanda Cristina de Lima Pinto Tavares (https://orcid.org/0000-0003-2118-7268) ${ }^{1}$
\end{abstract}

${ }^{1}$ Centro de Ciências da Saúde, Universidade Federal de Pernambuco. Av. Prof. Moraes Rego 1.235 , Cidade Universitária. 50670-901 Recife PE Brasil. marialaurenc@hotmail.com ${ }^{2}$ Centro Acadêmico de Vitória, Universidade Federal de Pernambuco. Vitória de Santo Antão PE Brasil.

\begin{abstract}
This study investigated the perception of above ideal body weight among adolescents in the city of Recife, Brazil, and its association with anthropometric profile and lifestyle. A cross-sectional study was conducted with a sample of 2480 adolescents from the "Study of Cardiovascular Risks in Adolescents." The conceptual model also assessed demographic and socioeconomic variables. Body weight perception was analyzed by asking the question: "In your opinion, your current body weight is ... ?" Answers included: below ideal, ideal and above ideal body weight. Statistics were identified using Poisson Regression analysis. The mean age was 14.7 years ( $S D=1.6), 53.7 \%$ were female, and $70.7 \%$ were eutrophic according to the body mass index/age (BMI/A). Perception of body weight was answered by $48.8 \%$ of the adolescents. $36.8 \%$ and $63.2 \%$ saw themselves as below and above their ideal weight, respectively. After adjustments, the variables associated with the perception of body weight above the ideal (outcome variable) were the female sex, age 12 to 14 years, mother's schooling $>8$ years, BMI/A in the ideal range, being postpubescent, having breakfast and meals with parents occasionally. Demographic, anthropometric and lifestyle variables were more likely to be associated with body weight perception above the ideal.
\end{abstract}

Key words Adolescent, Perception of weight, Body mass index
Resumo O objetivo da pesquisa foi analisar a percepção do peso corporal acima do ideal em adolescentes de Recife/PE e sua associação com o perfil antropométrico e estilo de vida. Trata-se de um estudo transversal com 2.480 escolares participantes do Estudo de Riscos Cardiovasculares em Adolescentes (Erica). O modelo conceitual também considerou variáveis demográficas e socioeconômicas. A percepção do peso foi analisada por meio da pergunta "Na sua opinião o seu peso atual é?", sendo as respostas: "abaixo do ideal", "ideal" $e$ "acima do ideal". As associações foram identificadas por meio da regressão de Poisson. A média da idade foi de 14,7 anos (DP = 1,6), 53,7\% eram do sexo feminino e $70,7 \%$ eram eutróficos, de acordo com o indice de massa corporal/idade (IMC/I). A percepção do peso foi respondida por $48,8 \%$ dos adolescentes. Desses, 36,8\% e 63,2\% se percebiam com baixo peso e peso acima do ideal, respectivamente. Após os ajustes, as variáveis associadas à percepção do peso acima do ideal foram: sexo feminino, idade entre 12 e 14 anos, escolaridade materna maior que oito anos, IMC/I de eutrofia e de excesso de peso, estágio pós-púbere e realização de café da manhã e refeição com os pais ocasionalmente. Portanto, variáveis demográficas, antropométricas e do estilo de vida se associaram à maior chance de percepção do peso corporal acima do ideal.

Palavras-chave Adolescente, Percepção de peso, Indice de massa corporal 


\section{Introdução}

A percepção do peso corporal reflete o modo como o indivíduo analisa seu próprio peso, as preocupações e a satisfação a ele relacionadas'. Nesse contexto, os fatores sociais, as influências culturais e a busca por um padrão de corpo ideal assumem papel de destaque, especialmente na adolescência ${ }^{2}$.

A literatura aponta que a percepção do peso corporal é melhor preditor na identificação dos adolescentes com comportamentos de controle de peso do que o diagnóstico real de excesso de peso $^{3}$. O crescimento e o desenvolvimento desses jovens tendem a ser prejudicados por atitudes inadequadas quando suas percepções não condizem com o corpo idealizado 4 . As preocupações com questões estéticas têm ocasionado alterações no comportamento alimentar, no estado nutricional e nos âmbitos social e psicológico desse público, interferindo assim em sua qualidade de vida $^{5}$.

De certo modo, as elevadas prevalências de excesso de peso contribuem para a insatisfação com o peso corporal. A Pesquisa Nacional de Saúde do Escolar (PeNSE), realizada pelo Instituto Brasileiro de Geografia e Estatística (IBGE) em 2009, com escolares do $9^{\circ}$ ano do ensino fundamental das capitais e do Distrito Federal, encontrou $16 \%$ de sobrepeso $(14,5 \%$ na rede pública e $21,4 \%$ na rede privada) e $7,2 \%$ de obesidade (6,5\% na rede pública e $9,9 \%$ na rede privada). No total, $23,2 \%$ dos escolares analisados apresentavam excesso de peso ${ }^{6}$.

A insatisfação com o corpo pode conduzir o indivíduo a adquirir comportamentos benéficos à saúde, como a alimentação saudável e a prática regular de atividade física ${ }^{7}$. Mas por outro lado, dependendo do grau de insatisfação, pode levar à adoção de comportamentos não condizentes com a saúde, tais como hábitos alimentares anormais (anorexia e bulimia nervosa) e práticas inadequadas de controle de peso $^{8}$. Nesse contexto, considerando a importância de se observar como os adolescentes percebem seus corpos, assim como os fatores que influenciam nesse processo, o presente estudo teve por objetivo analisar a percepção do peso corporal acima do ideal em adolescentes de 12 a 17 anos residentes na cidade de Recife/PE e sua associação com o perfil antropométrico e o estilo de vida.

\section{Métodos}

\section{Desenho e população do estudo}

Trata-se de um estudo transversal de base escolar realizado com adolescentes matriculados nos turnos da manhã e da tarde em escolas públicas e privadas da cidade do Recife, capital do estado de Pernambuco. Os dados foram coletados entre outubro de 2013 e maio de 2014, com interrupção das atividades nos meses de dezembro e janeiro em virtude do recesso escolar.

Os estudantes incluídos nesta investigação fazem parte do Estudo de Riscos Cardiovasculares em Adolescentes (Erica), uma pesquisa multicêntrica nacional que teve por objetivo principal estimar a prevalência de fatores associados ao risco cardiovascular em adolescentes?.

Neste trabalho foram avaliados 2.480 adolescentes, uma amostra representativa para uma estimativa de 142.421 escolares de 12 a 17 anos. A população foi composta por um maior percentual de estudantes de escolas públicas $(71,2 \%)$, sendo $53,2 \%$ do ensino fundamental.

\section{Amostragem}

A amostra foi do tipo estratificada, com três estágios de seleção: escola, turma e alunos, selecionados com probabilidade proporcional ao tamanho. Nas escolas escolhidas, foi realizado um levantamento das turmas e dos alunos das séries consideradas, para permitir a seleção de três turmas por escola. Naquela selecionadas, todos os alunos foram convidados a participar do estudo. Detalhes acerca da amostragem do Erica estão disponíveis em trabalhos já publicados ${ }^{9,10}$.

Como a pesquisa nacional gerou dados representativos para o país, as regiões e as capitais, optou-se, para esta investigação, pela avaliação de uma subamostra da população total analisada pelo Erica, sendo incluídos adolescentes que estavam matriculados nos turnos da manhã e tarde de 39 escolas públicas e privadas da cidade do Recife, obtendo-se uma amostra de 2.480 adolescentes.

\section{Variáveis do estudo, método de coleta e análise dos dados}

As variáveis incluídas neste estudo foram: percepção do peso corporal, sexo, idade, perfil socioeconômico e demográfico, peso corporal, altura, índice de massa corporal/idade (IMC/I), circunferência da cintura (CC), relação cintura/ 
estatura (RCEst), hábitos alimentares, nível de atividade física, estágios de maturação sexual, tabagismo e consumo de álcool.

\section{Percepção do peso corporal}

Para obtenção das informações sobre a percepção do peso corporal foram aplicados questionários a partir de um coletor eletrônico de dados autopreenchível, o Personal Digital Assistant (PDA). A percepção do peso corporal acima do ideal foi a variável dependente deste estudo, e para sua constituição foi utilizada a seguinte pergunta: "Na sua opinião o seu peso atual é?" Sendo classificada em "abaixo do ideal", "ideal", "acima" e "muito acima do ideal". Para fins de análise estatística, as opções "acima" e "muito acima do ideal” foram agrupadas em uma única opção, constituindo a categoria "acima do ideal".

Quando a percepção do peso relatada pelo adolescente era discrepante do perfil antropométrico real, ele era classificado com percepção inadequada do peso corporal. Padrões semelhantes de classificação são rotineiramente utilizados em pesquisas com esse mesmo propósito ${ }^{2,11,12}$.

\section{Perfil socioeconômico e demográfico}

As variáveis demográficas e socioeconômicas dos participantes foram obtidas segundo recomendações do IBGE $^{13}$, sendo coletadas informações relativas a sexo, idade, cor da pele e escolaridade materna. Em adição, os indivíduos foram classificados de acordo com a classe econômica, conforme os critérios da Associação Brasileira de Empresas de Pesquisa (ABEP) $)^{14}$, que divide as classes em alta (subcategorias A1, A2), média (subcategorias B1, B2) e baixa (subcategorias C, D e E). A ABEP leva em consideração a posse de bens de consumo conjuntamente com o grau de instrução do chefe da família. Com o total de pontos obtidos, classifica-se os indivíduos nas classes citadas. Para efeitos de análise, a idade cronológica dos adolescentes foi categorizada em "12-14" e "15-17" anos e a escolaridade materna em " $\leq 8$ anos" $\mathrm{e}$ " $>8$ anos de estudo".

\section{Perfil antropométrico}

Foram obtidas medidas de peso, altura e CC. O peso foi auferido em balança eletrônica Líder com capacidade de até 200 $\mathrm{kg}$ e variação de 50 g. A altura foi medida utilizando-se estadiômetro portátil e desmon- tável da marca Alturexata, com variação de $0,1 \mathrm{~cm}$ e campo de uso de até $213 \mathrm{~cm}$. As medições foram feitas em duplicata para fins de controle de qualidade. Foi admitida uma variação máxima de $0,5 \mathrm{~cm}$ entre as duas medições. Para as medidas de peso e altura, os adolescentes estavam descalços, usando roupas leves e em posição ortostática (no plano horizontal de Frankfurt) ${ }^{15}$.

Para a determinação do perfil antropométrico foi utilizado o software WHO AnthroPlus (2007), classificando os adolescentes pelo IMC/I, expresso segundo os valores em escore-Z. Para a classificação foram adotadas as curvas de referência da Organização Mundial de Saúde (OMS) ${ }^{16}$. Os pontos de corte adotados para categorização dos resultados foram: escore- $Z<-2$ (baixo peso); escore- $Z \geq-2$ e $\leq 1$ (eutrofia); escore- $Z>1$ e $\leq$ 2 (sobrepeso); e escore- $Z>2$ (obesidade). $\mathrm{Na}$ análise preliminar dos dados não foi evidenciado nenhum caso de obesidade severa, e entre os obesos a média no IMC/I ficou próxima dos limites inferiores. Ou seja, a grande maioria dos casos se concentrou na faixa próxima ao diagnóstico de obesidade. Assim, para fins de análise multivariada, as opções "sobrepeso" e "obesidade" foram agrupadas e deram origem à categoria "excesso de peso". Dessa forma, a variável IMC/I passou a ser constituída por três categorias: "baixo peso", "eutrofia" e "excesso de peso".

A mensuração da CC foi realizada com uma fita métrica inextensível da marca Sanny de 150 $\mathrm{cm}$ e variação de $0,1 \mathrm{~cm}$, estando o adolescente em posição ereta, com o abdômen relaxado, braços ao longo do corpo, pés juntos e com o peso dividido entre ambas as pernas. A fita foi colocada horizontalmente no ponto médio entre a borda inferior da última costela e a crista ilíaca ${ }^{15}$. As medidas foram feitas em duplicata, para fins de controle de qualidade. Foi permitida uma variação máxima de $1 \mathrm{~cm}$ entre as duas medições.

Os pontos de corte utilizados para o diagnóstico da obesidade abdominal a partir da CC foram os recomendados pela International Diabetes Federation ${ }^{17}$, que identifica como obesidade abdominal CC $\geq$ percentil 90 . Com as medidas da CC e da estatura foi realizado o cálculo da razão cintura/estatura (RCEst), estabelecendo-se como ponto de corte para obesidade abdominal valores iguais ou superiores a $0,5^{18}$. Para análise estatística, com as variáveis de CC e RCEst, que se propõem ao diagnóstico de obesidade abdominal, foram formadas duas categorias: "sem risco" e "com risco" para obesidade abdominal. 


\section{Hábito alimentar e tempo de tela}

O bloco de hábitos alimentares envolveu perguntas como realização do café da manhã, companhia dos pais ou responsáveis nas refeições (almoço e/ou jantar), refeições assistindo à TV e consumo de adoçante ou produto light/ diet. Todas as variáveis citadas tiveram as seguintes opções de resposta: "nunca/não", “às vezes” e "sempre".

A variável tempo de tela em frente à TV e ao computador foi categorizada em: " $\leq 2$ horas de tela por dia" e " $>2$ horas de tela por dia", tempo de tela considerado excessivo pela American Academy of Pediatrics ${ }^{19}$.

\section{Estágio de maturação sexual}

A avaliação da maturação sexual foi realizada por meio da auto avaliação com as pranchas de Tanner $(1962)^{20}$, técnica já validada para estudos populacionais $^{21,22}$. Solicitou-se que as adolescentes identificassem o estágio de desenvolvimento das mamas (M1, M2, M3, M4 e M5) que mais se assemelhavam aos seus corpos. Procedimento semelhante ocorreu com os meninos, aos quais foi requisitado que identificassem o estágio de desenvolvimento da genitália externa (G1, G2, G3, G4 e G5), avaliados de acordo com tamanho, forma e características.

O desenvolvimento dos pelos pubianos ( $\mathrm{P} 1$, P2, P3, P4 e P5) foi avaliado em ambos os sexos, conforme a quantidade, distribuição e características. Consideramos como pré-púberes as meninas e os meninos que se encontravam no estágio M1 e G1 do estágio de maturação sexual, como púberes aqueles que estavam no estágio M2, M3, M4, G2, G3 e G4, e no final da maturação sexual os que se encontravam no estágios M5 e G5 ${ }^{20}$. Para efeito de análise, os estágios de maturação pré-púbere e púbere foram combinados, formando uma única categoria, permanecendo as classificações “púberes” e "pós-púberes”.

\section{Nível de atividade física}

A fim de mensurar o nível de atividade física dos adolescentes, foi utilizada uma versão adaptada do Self-Administered Physical Activity Checklist $^{23}$, que consiste em uma lista de 24 modalidades e permite que o adolescente informe a frequência (dias) e o tempo (horas e minutos) que praticou alguma das atividades listadas na última semana. A determinação do nível de atividade física se deu de acordo com o somatório do produto do tempo despendido em cada uma das atividades e as respectivas frequências de prática. Os adolescentes que não acumularam pelo menos $300 \mathrm{~min}$./semana física foram considerados inativos $^{24}$.

\section{Tabagismo e consumo de álcool}

As informações sobre tabagismo foram obtidas realizando-se a seguinte pergunta ao adolescente: "Você fuma atualmente?" Com as opções de resposta "sim" ou "não". Quanto ao consumo de álcool, realizou-se o questionamento sobre o uso de bebida alcoólica nos últimos 30 dias (tomando como base a data da entrevista). Para efeito de análise, foram formadas as categorias de resposta "sim" ou "não".

\section{Plano de análise}

A associação entre as variáveis dependentes e independentes foi analisada por meio do teste qui-quadrado de Pearson. Como medida de associação foi utilizada a razão de prevalência (RP) com respectivo intervalo de confiança de 95\% (IC 95\%). As associações que apresentaram $\mathrm{p}<$ 0,20 na análise bruta foram submetidas à análise multivariada para controlar quaisquer potenciais fatores de confusão. As RPs e seus IC 95\% foram calculados por meio da regressão de Poisson com ajuste de variância robusta, tanto para a análise bruta quanto para a ajustada. O nível de significância estatística para as variáveis adotado no modelo final foi fixado em $\mathrm{p}<0,05$.

A amostra do Erica possui um delineamento complexo, visto que aplica estratificação e conglomeração e probabilidades desiguais em seus estágios de seleção ${ }^{9}$. Com isso, as análises estatísticas foram realizadas no software Stata versão 14.0 (StataCorp LP, College Station, Estados Unidos), programa estatístico que ajusta o delineamento amostral complexo a partir do módulo Survey.

\section{Aspectos éticos}

Em nível local, a presente pesquisa possui a aprovação do Comitê de Ética em Pesquisa da Universidade Federal de Pernambuco (CEP/ UFPE), em obediência à Resolução do Conselho Nacional de Saúde n466/12. Os adolescentes e seus responsáveis foram esclarecidos sobre todos os procedimentos, riscos e benefícios da pesquisa, sendo a participação voluntária. Os adolescentes assinaram um termo de assentimento livre 
Estre os adolescentes avaliados, 1.331 eram do sexo feminino $(53,7 \%), 53,9 \%$ apresentavam faixa etária entre 15-17 anos (a média da idade foi de 14,7 anos, DP $=1,6$ ) e $32,4 \%$ foram classificados no estágio pós-púbere de maturação sexual. Houve predomínio de escolares de classe econômica média $(48,2 \%)$ e baixa $(41,8 \%)$ cujas mães apresentavam mais de oito anos de escolaridade (70,1\%). Entre os estudantes, 1.367 (60,6\%) tinham um tempo de tela superior a duas horas/ dia (Tabela 1).

Um total de 1.219 adolescentes fizeram referência à percepção do peso corporal, sendo que nenhum deles se percebeu com peso adequado. Ou seja, 55,4\% e 7,8\%, respectivamente, perceberam-se acima e muito acima do peso, e 36,8\% se classificaram como baixo peso. Destaca-se que 1.261 adolescentes não responderam à essa questão. Por outro lado, quanto ao diagnóstico pelo IMC/I, 70,7\% estavam na categoria de eutrofia. Os adolescentes com sobrepeso e obesidade corresponderam a $16,1 \%$ e 9,3\%, respectivamente. Quanto à obesidade abdominal, diagnosticada pela CC e RCEst, a frequência foi de 10,4\%. No que se refere ao estilo de vida, $54,4 \%$ foram classificados como inativos, 21,6\% manifestaram consumir álcool e 1,6\% era fumante (Tabela 1).

$\mathrm{O}$ padrão de ocorrência de perdas quanto ao questionamento sobre a percepção do peso corporal foi avaliado a partir da comparação de algumas variáveis (socioeconômicas, demográficas, do estilo de vida e antropométricas) entre os adolescentes que não responderam (1.261) e responderam (1.219) a esse questionamento. Foi verificada maior ausência de resposta entre os meninos, os filhos de mães com maior escolaridade, os com perfil antropométrico adequado, mais ativos fisicamente, com maior tempo de tela e sem relato de consumo de álcool (resultados não expressos em tabela).

As tabelas 2 e 3 trazem o resultado da análise bivariada por meio do teste do qui-quadrado de Pearson. As variáveis associadas à percepção do peso corporal acima do ideal foram: sexo feminino, faixa etária de 12 a 14 anos, escolaridade materna maior que oito anos, classe econômica média e alta, IMC/I na faixa de eutrofia e excesso de peso, CC e RCEst na faixa de risco, pós-pú-

bere, não tomar café da manhã, ocasionalmente realizar as refeições com os pais, sempre utilizar adoçante/produto light/diet e o consumo de bebida alcoólica.

Após a regressão de Poisson, análise multivariada para controle de possíveis fatores de confusão, as variáveis que permaneceram independentemente associadas à percepção acima do peso ideal foram: sexo feminino $(\mathrm{RP}=1,16$; IC $95 \%=1,01-1,34)$, faixa etária de 12 a 14 anos (RP =1,30; IC 95\% = 1,15-1,46), estágio pós-púbere de maturação sexual ( $R P=1,17$; IC 95\% = 1,05$1,32)$, escolaridade materna superior a oito anos $(\mathrm{RP}=1,20 ; \mathrm{IC} 95 \%=1,01-1,46)$ e o fato de "às vezes" o adolescente tomar café da manhã ( $\mathrm{RP}=$ 1,14 ; IC 95\% = 0,99-1,32) e somente "às vezes" fazer as refeições em companhia dos pais ( $\mathrm{RP}=$ 1,35; IC 95\% = 1,05-1,73) (Tabela 4).

Quanto ao perfil antropométrico determinado pelo IMC/I, adolescentes com diagnóstico de excesso de peso tiveram 13,1 vezes mais chances de se perceber acima do peso corporal ideal. A probabilidade de se perceber dessa forma foi muito elevada até mesmo na categoria de eutrofia $(\mathrm{RP}=5,73$; IC 95\% = 1,69-19,4). Por outro lado, a obesidade abdominal identificada pela CC e pela RCEst não se mostrou independentemente associada à percepção do peso corporal acima do ideal (Tabela 4).

\section{Discussão}

Os achados do presente estudo sugerem a existência de associação entre a percepção do peso corporal acima do ideal e os fatores sociodemográficos, antropométricos e do estilo de vida de adolescentes de 12 a 17 anos da cidade de Recife.

Um percentual significativo $(25,4 \%)$ de adolescentes foi diagnosticado com excesso de peso, assemelhando-se aos resultados observados na PeNSE realizada em 2015, que evidenciou excesso de peso em $21,7 \%$ dos adolescentes da região Nordeste ${ }^{4}$.

O sobrepeso e a obesidade permanecem um desafio constante para a saúde pública ${ }^{4}$. Os prejuízos físicos e emocionais advindos dessas condições podem repercutir ao longo da vida do indivíduo ${ }^{25}$. Além disso, jovens com excesso de peso tendem a apresentar maior insatisfação corporal, pelo desejo em atender às representações sociais de padrão de beleza.

$\mathrm{Na}$ presente pesquisa, houve elevada prevalência de insatisfação com o peso corporal. Destaca-se que, entre os $48,8 \%$ dos adolescentes que 
Tabela 1. Características socioeconômicas, demográficas, de estilo de vida, antropométricas e percepção do peso corporal de adolescentes de 12-17 anos de idade em Recife - PE, 2013-2014.

\begin{tabular}{|c|c|c|c|c|}
\hline Variáveis & ${ }^{\square}$ Obs (2.480) & $\diamond_{\text {nEsti }}(142.421)$ & $\%$ & ${ }^{*} \mathrm{IC} 95 \%$ \\
\hline \multicolumn{5}{|l|}{ Sexo } \\
\hline Masculino & 1.149 & 72.286 & 46,3 & $44,4-48,3$ \\
\hline Feminino & 1.331 & 70.135 & 53,7 & $51,7-55,6$ \\
\hline \multicolumn{5}{|l|}{ Faixa etária (anos) } \\
\hline $12-14$ & 1.143 & 73.851 & 46,1 & $44,1-48,1$ \\
\hline $15-17$ & 1.337 & 68.570 & 53,9 & $51,9-55,9$ \\
\hline \multicolumn{5}{|l|}{ Maturação sexual' } \\
\hline Púberes & 1.670 & 98.630 & 67,6 & $65,6-69,4$ \\
\hline Pós-púberes & 802 & 43.789 & 32,4 & $30,6-34,3$ \\
\hline \multicolumn{5}{|l|}{ Escolaridade materna ${ }^{\circ}$} \\
\hline$\leq 8$ anos & 554 & 47.053 & 29,9 & $27,8-32,0$ \\
\hline$>8$ anos & 1.300 & 95.366 & 70,1 & $67,9-72,2$ \\
\hline \multicolumn{5}{|l|}{ Classe Econômica ${ }^{\circ}$} \\
\hline Alta & 170 & 12.294 & 10,0 & $8,6-11,5$ \\
\hline Média & 823 & 67.920 & 48,2 & $45,8-50,6$ \\
\hline Baixa & 713 & 62.207 & 41,8 & $39,4-44,2$ \\
\hline \multicolumn{5}{|c|}{ Percepção do peso corporal' $(\mathrm{n}=1.219)$} \\
\hline Baixo Peso & 448 & 55.144 & 36,8 & $34,0-39,5$ \\
\hline Sobrepeso & 676 & 74.617 & 55,4 & $52,6-58,3$ \\
\hline Obesidade & 95 & 12.660 & 7,8 & $6,4-9,5$ \\
\hline \multicolumn{5}{|l|}{ IMC/Idade ${ }^{o}$} \\
\hline Baixo peso & 97 & 5.652 & 3,9 & $3,2-4,8$ \\
\hline Eutrofia & 1.752 & 99.805 & 70,7 & $68,8-72,4$ \\
\hline Sobrepeso & 400 & 22.359 & 16,1 & $14,7-17,6$ \\
\hline Obesidade & 231 & 14.605 & 9,3 & $8,2-10,5$ \\
\hline \multicolumn{5}{|l|}{$\mathrm{CC}^{* *}$} \\
\hline Sem risco & 2.222 & 126.846 & 89,6 & $88,3-90,8$ \\
\hline Com risco & 258 & 15.575 & 10,4 & $9,2-11,7$ \\
\hline \multicolumn{5}{|l|}{ RCEst $^{* * *}$} \\
\hline Sem risco & 2.222 & 122.359 & 89,6 & $88,3-90,8$ \\
\hline Com risco & 258 & 20.062 & 10,4 & $9,2-11,7$ \\
\hline \multicolumn{5}{|c|}{ Nível de atividade física } \\
\hline Ativos & 1.130 & 67.276 & 45,6 & $43,6-47,6$ \\
\hline Inativos & 1.350 & 75.145 & 54,4 & $52,4-56,4$ \\
\hline \multicolumn{5}{|l|}{ Tempo de tela } \\
\hline$\leq 2$ horas/dia & 870 & 56.118 & 39,4 & $36,3-42,6$ \\
\hline$>2$ horas/dia & 1.367 & 86.302 & 60,6 & $57,4-63,7$ \\
\hline \multicolumn{5}{|l|}{ Fuma } \\
\hline Sim & 39 & 2.559 & 1,6 & $1,1-2,2$ \\
\hline Não & 2.441 & 139.862 & 98,4 & $97,8-98,9$ \\
\hline \multicolumn{5}{|l|}{ Bebe } \\
\hline Não & 1.823 & 113.847 & 78,4 & $71,7-75,2$ \\
\hline Sim & 502 & 28.574 & 21,6 & $18,7-21,9$ \\
\hline
\end{tabular}

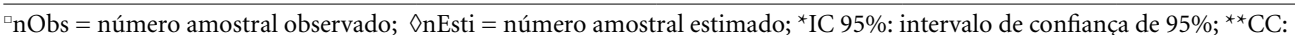
circunferência da cintura; ${ }^{* *}$ RCEst: relação cintura-estatura; ${ }^{*} \mathrm{IMC} / \mathrm{I}$ : índice de massa corporal para idade; $\bullet$ Nível de atividade física: ativos $\geq 300 \mathrm{~min} / \mathrm{sem}$, inativos $<300 \mathrm{~min} / \mathrm{sem;} \mathrm{'o} \mathrm{número} \mathrm{total} \mathrm{de} \mathrm{indivíduos} \mathrm{é} \mathrm{diferente} \mathrm{em} \mathrm{razão} \mathrm{do} \mathrm{número} \mathrm{de}$ respondentes.

Fonte: Elaborado pelos autores. 
Tabela 2. Distribuição dos estudantes de ambos os sexos com idade entre 12-17 anos segundo percepção do peso corporal e de variáveis demográficas, socioeconômicas, antropométricas e do estágio de maturação sexual, Recife - PE, 2013-2014.

\begin{tabular}{|c|c|c|c|c|c|c|c|c|c|}
\hline \multirow{3}{*}{ Variáveis } & \multicolumn{9}{|c|}{ Percepção do peso corporal acima do ideal } \\
\hline & \multicolumn{4}{|c|}{ Não $(\mathrm{n}=448)$} & \multicolumn{4}{|c|}{$\operatorname{Sim}(n=771)$} & \multirow[t]{2}{*}{${ }^{*} p$} \\
\hline & ${ }^{\square}$ nObs & $\diamond_{\text {nEsti }}$ & $\%$ & ${ }^{*} \mathrm{IC} 95 \%$ & ${ }^{\square}$ nObs & $\vartheta_{\text {nEsti }}$ & $\%$ & ${ }^{*} \mathrm{IC} 95 \%$ & \\
\hline \multicolumn{10}{|l|}{ Sexo } \\
\hline Masculino & 215 & 31.878 & 44,1 & $37,8-50,5$ & 275 & 40.407 & 55,9 & $49,4-62,2$ & 0,007 \\
\hline Feminino & 233 & 23.265 & 33,2 & $28,3-38,4$ & 496 & 46.869 & 66,8 & $61,6-71,7$ & \\
\hline \multicolumn{10}{|l|}{ Faixa etária (anos) } \\
\hline $12-14$ & 159 & 23.409 & 31,7 & $26,2-37,8$ & 378 & 50.441 & 68,3 & $62,24-73,8$ & $<0,001$ \\
\hline $15-17$ & 289 & 31.734 & 46,3 & $41,2-51,4$ & 393 & 36.835 & 53,7 & $48,59-58,77$ & \\
\hline \multicolumn{10}{|l|}{ Escolaridade materna } \\
\hline$\leq 8$ anos & 120 & 21.250 & 49,2 & $40,2-58,2$ & 139 & 21.949 & 50,8 & $41,8-59,8$ & 0,006 \\
\hline$>8$ anos & 227 & 35.394 & 35,7 & $31,3-40,3$ & 453 & 63.825 & 64,3 & $59,7-68,7$ & \\
\hline \multicolumn{10}{|l|}{ Classe econômica } \\
\hline Alta & 27 & 3.693 & 29,3 & $20,9-39,3$ & 70 & 8.926 & 70,7 & $60,7-79,1$ & \\
\hline Média & 138 & 23.715 & 35,3 & $30,5-40,5$ & 260 & 43.370 & 64,7 & $59,5-69,5$ & 0,023 \\
\hline Baixa & 147 & 28.047 & 44,7 & $36,8-52,9$ & 210 & 34.666 & 55,3 & $47,1-63,2$ & \\
\hline \multicolumn{10}{|l|}{$\mathrm{IMC} / \mathrm{I}^{\circ}$} \\
\hline Baixo peso & 58 & 7.080 & 96,1 & $85,5-99,0$ & 2 & 285 & 3,9 & $0,9-14,5$ & \\
\hline Eutrofia & 369 & 45.487 & 57,3 & $52,5-61,9$ & 316 & 33.905 & 42,7 & $38,0-47,5$ & $<0,001$ \\
\hline Sobrepeso & 16 & 1.857 & 6,2 & $3,8-10,1$ & 260 & 28.017 & 93,8 & $89,9-96,2$ & \\
\hline Obesidade & 5 & 718 & 2,8 & $0,9-8,0$ & 193 & 25.069 & 97,2 & $91,9-99,1$ & \\
\hline \multicolumn{10}{|l|}{$\mathrm{CC}^{\star *}$} \\
\hline Normal & 442 & 54.323 & 47,1 & $42,6-51,7$ & 562 & 60.923 & 52,9 & $48,3-57,4$ & $<0,001$ \\
\hline Elevada & 6 & 820 & 3,0 & $1,7-7,6$ & 209 & 26.352 & 97,0 & $92,4-98,8$ & \\
\hline \multicolumn{10}{|l|}{ RCEst $^{* * *}$} \\
\hline Normal & 441 & 54.168 & 50,1 & $45,5-54,8$ & 499 & 53.869 & 49,9 & $45,2-54,5$ & $<0,001$ \\
\hline Obes. abdominal & 7 & 975 & 2,8 & $1,2-6,7$ & 272 & 33.407 & 97,2 & $93,3-98,8$ & \\
\hline \multicolumn{10}{|l|}{ Maturação sexual } \\
\hline Púberes & 315 & 40.111 & 41,6 & $36,6-46,8$ & 472 & 56.303 & 58,4 & $53,2-63,4$ & 0,012 \\
\hline Pós-púberes & 133 & 15.228 & 33,1 & $27,9-38,7$ & 294 & 30.777 & 66,9 & $61,3-72,0$ & \\
\hline
\end{tabular}

${ }^{\square}$ Obs $=$ número amostral observado; $\diamond$ nEsti $=$ número amostral estimado; ${ }^{\star}$ IC 95\%: intervalo de confiança; ${ }^{\star \star}$ CC: circunferência da cintura; ${ }^{\star * \star}$ RCEst: relação cintura-estatura; ${ }^{\star} \mathrm{p}=$ teste qui-quadrado; ${ }^{\circ} \mathrm{IMC} / \mathrm{I}$ : índice de massa corporal para idade.

Fonte: Elaborado pelos autores.

responderam sobre suas percepções, nenhum relatou peso adequado. Ou seja, esse percentual se refere a quem se percebia abaixo ou acima do peso, apesar da eutrofia pelo IMC/I ter sido em torno de $70,0 \%$.

Vale a pena salientar que, entre os estudantes que não responderam, houve maior frequência do sexo masculino, eutróficos pelo IMC/I e pela CC. Ou seja, talvez esses adolescentes não tenham dado a devida importância a esse questionamento por não apresentarem problemas com a imagem corporal.

Os achados encontrados entre os 1.219 adolescentes que responderam, trazem um alerta não só para a percepção acima do ideal, mas também abaixo, pois $36,8 \%$ se percebiam com baixo peso quando apenas 3,9\% apresentavam essa condição pelo IMC/I.

De maneira geral, escolares de ambos os sexos apresentaram algum grau de distorção da imagem corporal. Entretanto, as meninas se perceberam mais acima do peso do que os meninos. Esse resultado é respaldado por inúmeras pesquisas com esse mesmo propósito ${ }^{2,8,11}$.

Em estudo prévio realizado em Santa Catarina com 1.051 adolescentes de 15 a 19 anos de ambos os sexos, $49,9 \%$ das garotas tinham uma percepção do peso acima de sua condição corporal ${ }^{8}$. Pode-se inferir que como as meninas sofrem mais pressão social do que meninos, elas apresentam mais dificuldades em aceitar seu corpo e maior probabilidade de perceber seu peso 
Tabela 3. Distribuição dos estudantes de ambos os sexos com idade entre 12-17 anos segundo percepção do peso corporal e variáveis do estilo de vida, Recife - PE, 2013-2014.

\begin{tabular}{|c|c|c|c|c|c|c|c|c|c|}
\hline \multirow{3}{*}{ Variáveis } & \multicolumn{9}{|c|}{ Percepção do peso corporal acima do ideal } \\
\hline & \multicolumn{4}{|c|}{ Não $(n=448)$} & \multicolumn{4}{|c|}{$\operatorname{Sim}(\mathbf{n}=771)$} & \multirow[t]{2}{*}{${ }^{*} \mathbf{p}$} \\
\hline & ${ }^{\square}$ nObs & $\diamond$ nEsti & $\%$ & ${ }^{*} \mathrm{IC} 95 \%$ & ${ }^{\square}$ nObs & $\diamond$ nEsti & $\%$ & ${ }^{*} \mathrm{IC} 95 \%$ & \\
\hline \multicolumn{10}{|l|}{ Toma café da manhã } \\
\hline Não & 74 & 8.793 & 28,7 & $22,4-36,0$ & 213 & 21.820 & 71,3 & $63,9-77,6$ & \\
\hline Às vezes & 151 & 17.146 & 35,9 & $30,3-42,1$ & 264 & 30.505 & 64,0 & $57,9-69,7$ & \multirow[t]{2}{*}{$<0,001$} \\
\hline Sempre & 223 & 29.203 & 45,5 & $39,9-51,3$ & 294 & 34.950 & 54,5 & $48,7-60,1$ & \\
\hline \multicolumn{10}{|l|}{ Refeições com os pais } \\
\hline Nunca & 67 & 7.150 & 47,3 & $38,6-56,3$ & 86 & 7.952 & 52,7 & $43,7-61,4$ & \multirow{3}{*}{0,023} \\
\hline Às vezes & 141 & 16.550 & 34,8 & $30,5-39,4$ & 270 & 30.988 & 65,2 & $60,6-69,5$ & \\
\hline Sempre & 240 & 31.443 & 39,4 & $34,1-44,9$ & 415 & 48.335 & 60,6 & $55,0-65,8$ & \\
\hline \multicolumn{10}{|c|}{ Refeições assistindo TV (telas) } \\
\hline Nunca & 35 & 4.235 & 28,4 & $20,5-37,9$ & 90 & 10.668 & 71,6 & $62,1-79,4$ & \multirow{3}{*}{0,163} \\
\hline Às vezes & 128 & 15.338 & 37,7 & $30,3-45,8$ & 232 & 25.303 & 62,2 & $54,2-69,7$ & \\
\hline Sempre & 285 & 35.569 & 40,9 & $34,9-47,2$ & 449 & 51.304 & 59,1 & $52,8-65,1$ & \\
\hline \multicolumn{10}{|c|}{ Uso de adoçante/produto light ou diet } \\
\hline Não & 339 & 44.860 & 41,7 & $36,6-47,0$ & 502 & 62.654 & 58,3 & $52,9-63,4$ & \multirow{3}{*}{0,018} \\
\hline Às vezes & 62 & 8.248 & 32,1 & $25,5-39,9$ & 151 & 17.280 & 67,7 & $60,0-74,5$ & \\
\hline Sempre & 15 & 2.281 & 24,3 & $14,2-38,5$ & 59 & 7.095 & 75,7 & $61,5-85,8$ & \\
\hline \multicolumn{10}{|c|}{ Tempo de tela (computador e TV) } \\
\hline$\leq 2 \mathrm{~h}$ por dia & 148 & 18.575 & 36,6 & $31,0-42,5$ & 254 & 32.208 & 63,4 & $57,5-68,9$ & \multirow[t]{2}{*}{0.5441} \\
\hline$>2$ horas por dia & 253 & 35.468 & 38,7 & $33,7-43,9$ & 447 & 56.168 & 61,3 & $56,0-66,3$ & \\
\hline \multicolumn{10}{|c|}{ Nível de atividade física } \\
\hline Ativos & 193 & 26.021 & 39,9 & $34,8-45,1$ & 323 & 39.262 & 60,1 & $54,9-65,2$ & \multirow[t]{2}{*}{0,481} \\
\hline Inativos & 255 & 29.122 & 37,7 & $32,6-43,2$ & 448 & 48.013 & 62,2 & $56,8-67,4$ & \\
\hline \multicolumn{10}{|l|}{ Fuma } \\
\hline Sim & 6 & 615 & 22,8 & $10,4-42,8$ & 17 & 2.085 & 77,2 & $57,2-89,6$ & \multirow[t]{2}{*}{0,096} \\
\hline Não & 442 & 54.528 & 39,0 & $34,8-43,4$ & 754 & 85.191 & 60,9 & $56,6-65,2$ & \\
\hline \multicolumn{10}{|l|}{ Bebe } \\
\hline Não & 323 & 40.273 & 36,3 & $32,5-40,3$ & 581 & 70.616 & 63,7 & $59,6-67,5$ & \multirow[t]{2}{*}{0,010} \\
\hline Sim & 109 & 14.520 & 46,0 & $38,5-53,8$ & 161 & 17.010 & 53,9 & $46,2-61,5$ & \\
\hline
\end{tabular}

Fonte: Elaborado pelos autores.

equivocadamente, situação que pode ser agravada com as mudanças corporais intrínsecas à puberdade.

Entre as meninas, a busca por um perfil corporal magro, muitas vezes difícil de ser atingido, é algo recorrente e consolidado na literatura. Conjectura-se que, em uma cultura em que a atratividade corporal está ligada à magreza, a procura pelo corpo idealizado e valorizado socialmente torna-se atitude sine qua non entre as adolescentes, a ponto de acarretar distorção da percepção corporal. As garotas muitas vezes se sentem acima do peso quando na realidade se encontram na faixa de normalidade ${ }^{26}$, situação averiguada no presente estudo.
Esta pesquisa constatou associação entre o fato de às vezes o adolescente realizar o café da manhã e o desfecho avaliado. Resultado semelhante foi corroborado em estudo prévio conduzido com 3.939 adolescentes japonesas, em que a omissão diária do café da manhã esteve relacionada com a superestimação do peso corporal ${ }^{27}$.

Branco e colaboradores ${ }^{2}$ apontaram uma relação significante entre a distorção da imagem corporal e a omissão do desjejum entre as meninas, sugerindo que os jovens insatisfeitos com seu corpo recorrem a tais meios para redução do consumo calórico em tentativa de perder peso. Pesquisa com estudantes de Caruaru/PE evidenciou chance 1,79 vez maior de insatisfação pelo 
Tabela 4. Razão de prevalência bruta e ajustada da percepção corporal acima do ideal em adolescentes segundo variáveis demográficas, socioeconômicas e antropométricas e estágio de maturação sexual e estilo de vida. Recife - PE, 2013-2014.

\begin{tabular}{|c|c|c|c|c|c|}
\hline \multirow{2}{*}{ Variáveis } & \multicolumn{4}{|c|}{ Percepção do peso corporal acima do ideal $(n=771)$} & \multirow[b]{2}{*}{$\diamond \mathrm{p}$} \\
\hline & -RP bruta & ${ }^{\star} \mathrm{IC}_{95 \%}$ & -RP ajustada & ${ }^{\star} \mathbf{I C}_{95 \%}$ & \\
\hline \multicolumn{6}{|l|}{ Sexo } \\
\hline Masculino & 1,00 & & 1,00 & & \\
\hline Feminino & 1,19 & $1,04-1,36$ & 1,16 & $1,01-1,34$ & 0,030 \\
\hline \multicolumn{6}{|l|}{ Faixa etária (anos) } \\
\hline $15-17$ & 1,00 & & 1,00 & & \\
\hline $12-14$ & 1,27 & $1,13-1,42$ & 1,30 & $1,15-1,46$ & $<0,001$ \\
\hline \multicolumn{6}{|l|}{ Escolaridade materna } \\
\hline$\leq 8$ anos & 1,00 & & 1,00 & & \\
\hline$>8$ anos & 1,26 & $1,05-1,52$ & 1,20 & $1,01-1,46$ & 0,048 \\
\hline \multicolumn{6}{|l|}{ Classe econômica } \\
\hline Baixa & 1,00 & & 1,00 & & \\
\hline Média & 1,17 & $1,01-1,35$ & 1,14 & $0,97-1,34$ & 0,092 \\
\hline Alta & 1,27 & $1,01-1,60$ & 1,22 & $0,98-1,53$ & 0,071 \\
\hline \multicolumn{6}{|l|}{$\mathrm{IMC} / \mathrm{I}^{\circ}$} \\
\hline Baixo peso & 1,00 & & 1,00 & & \\
\hline Eutrofia & 11,0 & $2,8-43,3$ & 5,73 & $1,69-19,4$ & 0,006 \\
\hline Excesso de peso & 7,78 & $7,17-8,45$ & 13,1 & $3,97-43,6$ & $<0,001$ \\
\hline \multicolumn{6}{|l|}{$\mathrm{CC}^{* *}$} \\
\hline Normal (sem risco) & 1,00 & & 1,00 & & \\
\hline Elevada (com risco) & 1,83 & $1,68-1,99$ & 1,09 & $0,95-1,26$ & 0,188 \\
\hline \multicolumn{6}{|l|}{ RCEst $^{* * *}$} \\
\hline Normal (sem risco) & 1,00 & & 1,00 & & \\
\hline Obesidade abdominal & 1,94 & $1,78-2,13$ & 0,99 & $0,91-1,08$ & 0,862 \\
\hline \multicolumn{6}{|l|}{ Maturação sexual } \\
\hline Púberes & 1,00 & & 1,00 & & \\
\hline Pós-púberes & 1,14 & $1,03-1,27$ & 1,17 & $1,05-1,32$ & 0,006 \\
\hline \multicolumn{6}{|l|}{ Toma café da manhã } \\
\hline Sempre & 1,00 & & 1,00 & & \\
\hline Às vezes & 1,17 & $1,02-1,34$ & 1,14 & $0,99-1,32$ & 0,006 \\
\hline Não & 1,30 & $1,17-1,45$ & 1,20 & $1,05-1,37$ & 0,067 \\
\hline \multicolumn{6}{|l|}{ Refeições com os pais } \\
\hline Nunca & 1,00 & & 1,00 & & \\
\hline Às vezes & 1,23 & $1,05-1,45$ & 1,35 & $1,05-1,73$ & 0,020 \\
\hline Sempre & 1,15 & $0,97-1,36$ & 1,25 & $0,99-1,57$ & 0,056 \\
\hline \multicolumn{6}{|l|}{ Refeições assistindo TV } \\
\hline Sempre & 1,00 & & 1,00 & & \\
\hline Às vezes & 1,05 & $0,89-1,24$ & 0,95 & $0,77-1,16$ & 0,636 \\
\hline Não & 1,21 & $1,03-1,42$ & 1,19 & $0,96-1,47$ & 0,108 \\
\hline \multicolumn{6}{|c|}{ Uso de adoçante/produto light ou diet } \\
\hline Não & 1,00 & & 1,00 & & \\
\hline Às vezes & 1,16 & $1,01-1,33$ & 1,04 & $0,89-1,22$ & 0,564 \\
\hline Sempre & 1,29 & $1,06-1,57$ & 1,16 & $0,89-1,51$ & 0,246 \\
\hline \multicolumn{6}{|l|}{ Fuma } \\
\hline não & 1,00 & & 1,00 & & \\
\hline $\operatorname{sim}$ & 1,26 & $1,01-1,57$ & 0,85 & $0,31-2,27$ & 0,740 \\
\hline \multicolumn{6}{|l|}{ Bebe } \\
\hline Sim & 1,00 & & 1,00 & & \\
\hline Não & 1,18 & $1,02-1,35$ & 1,17 & $0,92-1,47$ & 0,186 \\
\hline
\end{tabular}

${ }^{\star}$ IC 95\%: intervalo de confiança de 95\%; •Razão de prevalência; ${ }^{* *}$ CC: circunferência da cintura; ${ }^{* *}$ RCEst: relação cinturaestatura; $\bullet$ p = teste qui-quadrado de Pearson; ${ }^{\circ} \mathrm{IMC} / \mathrm{I}$ : índice de massa corporal para idade.

Fonte: Elaborado pelos autores. 
excesso de peso naqueles que não consumiam as três refeições diárias ${ }^{29}$.

Vliet, Gustafsson e Nelson ${ }^{30}$ constataram que tanto o IMC/I quanto a CC estiveram associados à menor frequência de consumo de café da manhã em meninas. Além disso, o risco de pular o café da manhã aumentou quando a percepção era de sobrepeso e a idade maior de 13 anos para ambos os sexos. Possivelmente, o aumento do peso e gordura abdominal são vistos como obstáculos para a aquisição do corpo idealizado, assim os jovens utilizam estratégias não condizentes com a saúde para controle do peso e alcance da forma física.

A realização de refeições (almoço e jantar) com os pais, bem como sua frequência, foi outra variável investigada. $\mathrm{O}$ fato de às vezes $\mathrm{o}$ adolescente fazer as refeições com seus pais esteve associada à percepção acima do ideal. Isso reflete, sobretudo, um quadro de diminuição das refeições em família devido à maior autonomia dos adolescentes nas escolhas alimentares, à influência dos pares e ao hábito de dietas, especialmente em meninas ${ }^{30}$.

É importante frisar que tanto o café da manhã quanto a realização de refeições com os pais constituem hábitos alimentares saudáveis que devem ser incentivados na população jovem. Por sua vez, o café da manhã é uma refeição crucial e contribui para um consumo alimentar saudável que perdurará até a idade adulta. Conjectura-se que esses aspectos atuem como estratégias de prevenção de sobrepeso e obesidade e propiciem uma percepção do peso corporal positiva pelos adolescentes.

Nesta pesquisa foi observado que os adolescentes no estágio pós-púbere de maturação sexual se percebiam mais acima do peso do que os púberes. Essa relação também é constatada na literatura ${ }^{31}$ e fundamentada pelas mudanças fisiológicas que ocorrem durante esse período.

Com o advento do desenvolvimento puberal, as meninas tendem ao ganho de tecido adiposo, não necessariamente apresentando excesso de peso, mas considerando as influências socioculturais, elas podem se perceber distantes do padrão de beleza amplamente valorizado, favorecendo a insatisfação e a percepção inadequada do peso corporal. Essas atitudes também são partilhadas pelos meninos, em particular quando se comparam com seus pares, mesmo sendo sua maturação coincidente com o ideal de beleza masculino ${ }^{12}$.

Os adolescentes na faixa etária de 12 a 14 anos se perceberam mais acima do peso em comparação com aqueles de 15 a 17 anos. Esperava-se que os escolares de maior idade superestimassem mais seu peso corporal, a exemplo do observado por Shin e $\mathrm{Nam}^{2}$ em estudo com adolescentes de 12 a 18 anos na Coréia do Sul, em que as meninas com maior idade tiveram maior probabilidade de superestimar a percepção do peso.

O estágio de maturação sexual talvez possa explicar esse resultado, uma vez que essa faixa etária corresponde ao estágio pré-púbere e púbere de maturação, em que meninos e meninas ainda irão iniciar ou estão no princípio do processo de maturidade e adentrarão o auge do crescimento, podendo não se perceber com o peso corporal adequado, o que aponta o quão influenciados os jovens são desde cedo pelos padrões de beleza contemporâneos ${ }^{12}$.

Nessa casuística, os adolescentes que tinham mães com mais de 8 anos de estudo tiveram mais chances de perceber seu peso acima do ideal (superestimação). Um estudo com 3.321 adolescentes coreanos trouxe resultados discrepantes, em que jovens cujas mães possuíam maior escolaridade tiveram maior probabilidade de subestimar seu peso corporal ${ }^{2}$. Por outro lado, na pesquisa de Pereira e colaboradores ${ }^{12}$, realizada em Vitória/ES, não foi constatada relação entre essas variáveis. Deve-se destacar, no entanto, a heterogeneidade socioeconômica entre os adolescentes brasileiros e coreanos, o que poderia ajudar a explicar a discordância entre os achados.

Esperava-se que um nível elevado de escolaridade se refletisse em maior consciência do peso devido ao melhor acesso a informações de saú$\mathrm{de}^{26}$. Em contrapartida, na classe mais escolarizada, as exigências quanto ao corpo para se atingir o padrão de beleza vislumbrado também podem se tornar maiores, contribuindo para distorção da percepção do peso, sendo uma possível explicação para o resultado observado.

O presente estudo apresenta algumas limitações metodológicas que devem ser ressaltadas, como o fato de ser um recorte de uma amostra complexa e de âmbito nacional, inviabilizando uma visão ampliada da percepção do peso corporal em adolescentes brasileiros. Também apresenta um delineamento do tipo transversal, não permitindo que se faça inferências de causa e efeito. Além disso, devemos salientar os possíveis vieses na coleta dos dados, tais como o viés de memória e a omissão de informações nas respostas fornecidas pelos adolescentes.

Em contrapartida, uma característica importante desta pesquisa é a investigação da percepção do peso corporal em uma amostra de base populacional. Embora o estudo não retrate dados 
nacionais, a iniciativa de um estudo regional permite a obtenção de informações inéditas que podem ser utilizadas como indicadores para comparações regionais, nacionais e internacionais.

A investigação desagregada permite conhecer características locais que podem não ser percebidas em unidades de análise nacionais. Em consonância com esse fato, a pesquisa possibilitou avaliar o quadro situacional específico da cidade do Recife/PE, buscando compreender melhor as peculiaridades da região, de forma a subsidiar políticas públicas locais para a resolução dos possíveis problemas encontrados.

Em síntese, a maioria dos adolescentes foi classificada com eutrofia $(70,7 \%)$, embora nenhum tenha se percebido com peso ideal. Jovens de ambos os sexos apresentaram algum grau de distorção da percepção corporal e variáveis demográficas, antropométricas e do estilo de vida se associaram à maior chance de percepção do peso corporal acima do ideal. Uma proporção expressiva de escolares se percebia com peso ina- dequado mesmo quando o diagnóstico era de eutrofia. Por outro lado, é importante destacar a taxa significativa de excesso de peso encontrada, sugerindo um alerta para ações que tenham como propósito a prevenção desse agravo e a promoção da saúde.

Por fim, no estado de Pernambuco, o estudo de Costa e colaboradores ${ }^{32}$, que avaliou a condição nutricional de mulheres de 10 a 49 anos em um intervalo de nove anos (1997-2006), observou que, a elevação do excesso de peso foi maior na faixa etária entre 10 e 19 anos, e algumas evidências apontam que o período de maior risco para incidência da obesidade é a transição entre a adolescência e as etapas precoces da vida adulta, em ambos os sexos e em vários grupos étnicos ${ }^{33}$. Desse modo, torna-se importante para o município avaliado ter conhecimento sobre a condição antropométrica de seus adolescentes, bem como a respeito de como esses jovens percebem o peso corporal, objetivando medidas de prevenção primária e secundária nesse subgrupo populacional.

\section{Colaboradores}

MJLN Carvalho participou do delineamento e planejamento do artigo, da análise, interpretação e discussão dos dados, bem como da redação e revisão final do manuscrito. PC Cabral e JS Oliveira participaram do delineamento do artigo, da orientação do trabalho, da análise e interpretação dos dados e da revisão crítica do manuscrito final. PIC Lira participou do direcionamento teórico-metodológico do trabalho e da revisão crítica do manuscrito final. MIS Andrade participou da análise e interpretação dos dados. VS Leal, LAS Sequeira-de-Andrade e FCLP Tavares contribuíram no direcionamento teórico do trabalho e na revisão crítica do manuscrito final.

\section{Referências}

1. Nunes MA, Olinto MTA, Barros FC, Camey S. Influência da percepção do peso e do índice de massa corporal nos comportamentos alimentares anormais. Braz J Psychiatry 2001; 23(1):21-27.

2. Shin A, Nam CM. Weight perception and its association with socio-demographic and health-related factors among Korean adolescents. BMC Public Health 2015; 15:1292.

3. Jones, N. Relationship of BMI and Weight Perception to Weight Controlling Behaviors in 9th-12th Graders in the United States [dissertação]. United States: University of Kentucky, College of Public Health; 2015.

4. Instituto Brasileiro de Geografia e Estatística (IBGE) Pesquisa nacional de saúde do escolar 2015. Rio de Janeiro: IBGE; 2016.

5. Paludo J, Dalpubel V. Imagem corporal e sua relação com o estado nutricional e a qualidade de vida de adolescentes de um município do interior do Rio Grande do Sul. Nutrire journal of the Brazilian Society of Food and Nutrition 2015; 40(1):1-9.

6. Instituto Brasileiro de Geografia e Estatística (IBGE) Pesquisa nacional de saúde do escolar 2009. Rio de Janeiro: IBGE; 2009.

7. Tribess S. Percepção da imagem corporal e fatores relacionados à saúde em idosas. [dissertação]. Florianópolis: Universidade Federal de Santa Catarina; 2006. 
8. Frank R., Claumann GS, Felden EPG, Silva DAS, Pelegrini A. Body weight perception and body weight controlbehaviors in adolescents. J Pediatr 2018; 94(1): 40-47.

9. Vasconcellos MTL, Silva PLN, Szklo M, Kuschnir MCC, Klein CH, Abreu GA, Barufaldi LA, Bloch KV. Sampling design for the Study of Cardiovascular Risks in Adolescents (ERICA). Cad Saude Publica 2015; 31(5):921-930.

10. Bloch KV, Szklo M, Kuschnir MC, Abreu GA, Barufaldi LA, Klein CH, Vasconcelos MT, Veiga GV, Figueiredo VC, Dias A, Moraes AJ, Souza AL, Oliveira AM, Schaan BD, Tavares BM, Oliveira CL, Cunha CF, Giannini DT, Belfort DR, Ribas DL, Santos EL, Leon EB, Fujimori E, Oliveira ER, Magliano ES, Vasconcelos FA, Azevedo GD, Brunken GS, Dias GM, Filho HR, Monteiro MI, Guimarães IC, Faria Neto JR, Oliveira JS, Carvalho KM, Gonçalves LG, Santos MM, Muniz PT, Jardim PC, Ferreira PA, Montenegro RM Jr, Gurgel RQ, Vianna RP, Vasconcelos SM, Matta SS, Martins SM, Goldberg TB, Silva TL. The Study of Cardiovascular Risk in Adolescents - ERICA: rationale, design and sample characteristics of a national survey examining cardiovascular risk factor profile in Brazilian adolescents. BMC public health 2015; 15(1):94.

11. Shirasawa T, Ochiai H, Nanri H, Nishimura R, Ohtsu T, Hoshino H, Tajima N, Kokaze A. The relationship between distorted body image and lifestyle among Japanese adolescents: a population-based study. Arch Public Health 2015; 73(1):32.

12. Pereira FN, Oliveira JR, Zöllner CC, Gambardella AMD. Percepção do peso corporal e fatores associados em estudantes. Rev Bras Crescimento Desenvolv Hum 2013; 23(2):170-176.

13. Instituto Brasileiro de Geografia e Estatística (IBGE). Household Budget Survey 2002-2003: analysis of household food availability and nutritional status in Brazil. Rio de Janeiro: IBGE; 2004.

14. Associação Brasileira de Empresas de Pesquisa (ABEP). Critério Brasil de avaliação econômica. São Paulo: ABEP; 2013.

15. Sistema de Vigilância Alimentar e Nutricional (SISVAN). Orientações para a coleta e análise de dados antropométricos em serviços de saúde. Brasília: SISVAN; 2011.

16. Onis M, Onyango AW, Borghi E, Siyam A, Nishida C, Siekmann J. Development of a WHO growth reference for school-ages children and adolescents. Bull World Health Org 2007; 85(9):660-667.

17. International Diabetes Federation (IDF). The IDF consensus: definition of the metabolic syndrome in children and adolescents. Brussels: IDF; 2007.

18. 18. Ashwell M, Hsieh SD. Six reasons why the waistto-height ratio is a rapid and effective global indicator for health risks of obesity and how its use could simplify the international public health message on obesity. Int J Food Sci Nutr 2005; 56(5):303-307.

19. American Academy of Pediatrics. Committee on Public Education. American Academy of Pediatrics: children, adolescents, and television. Pediatrics 2001; 107(2):423-426.

20. Tanner JM. Growth at adolescence. Oxford: Blackwell Scientific Publications; 1962.

21. Duke PM, Litt IF, Gross RT. Adolescent's self-assessment of sexual maturation. Pediatrics 1980; 66(6):918-920.
22. Saito MI. Maturação sexual: auto-avaliação do adolescente. Pediatr 1984; 6:111-115.

23. Farias Júnior JC, Lopes, AS, Mota J, Santos MP, Ribeiro JC, Hallal PC. Validade e reprodutibilidade de um questionário para medida de atividade física em adolescentes: uma adaptação do Self-Administered Physical Activity Checklist. Rev Bras Epidemiol 2012; 15(1):198-210.

24. Guedes DP, Lopes CC, Guedes JERP. Reproducibility and validity of International Physical Activity Questionnaire in adolescents. Rev bras med esporte 2005; 11:151-158.

25. Baker KD, Loughman A, Spencer SJ, Reichelt AC. The impact of obesity and hypercaloric diet consumption on anxiety and emotional behavior across the lifespan. Neurosci Biobehav Rev 2017; 83:173-182.

26. Frayon S, Cherrier S, Cavaloc Y, Wattelez G, Touitou A, Zongo P, Yacef K, Caillaud C, Lerrant Y, Galy O. Misperception of weight status in the pacific: preliminary findings in rural and urban 11- to 16-year-olds of New Caledonia. BMC Public Health 2017; 17(1):25.

27. Mori K, Sekine M, Yamagami T, Kagamimori S. Relationship between body image and lifestyle factors in Japanese adolescent girls. Pediatr Int 2009; 51(4):507513.

28. Branco LM, Almeida EC, Passos MAZ, Piano A, Cintra IP, Fisberg M. Percepção corporal influência no consumo do café da manhã de adolescentes? Health in review 2007; 9:15-21.

29. Santos EMC, Tassitano RM, Nascimento WMF, Petribú MMV, Cabral PC. Satisfação com o peso corporal e fatores associados em estudantes do ensino médio. Rev Paul Pediatr 2011; 29(2):214-223.

30. Vliet JSV, Gustafsson PA, Nelson N. Feeling 'too fat' rather than being 'too fat' increases unhealthy eating habits among adolescents even in boys. Food Nutr Res 2016; 60:29530.

31. Romanholo RA, Belo C, Baia FC, Pereira JE, Lucena ARN, Prestes J, Almeida FM. Maturação biológica e imagem corporal; sua relação com o sobrepeso/obesidade em escolares de Cacoal, Rondônia. J Health Bio Sci 2017; 5(3):234-240.

32. Costa EC, Lira PIC, Oliveira JS, Menezes RCE, Tavares FCLP, Batista Filho M. Overweight change and associated factors in women aged 10 to 49 years from Pernambuco, Brazilian Notheast. Rev Nutr 2014; 27(5): 513-524.

33. Gordon-Larsen P, Adair LS, Nelson MC, Popkin BM. Five-year obesity incidence in the transition period between adolescence and adulthood: the National Longitudinal Study of Adolescent Health. Am J Clin Nutr 2004; 80(3):569-575.

Artigo apresentado em 20/07/2019

Aprovado em 01/04/2020

Versão final apresentada em 03/04/2020

Editores-chefes: Romeu Gomes, Antônio Augusto Moura da Silva 